\title{
Variable Temperature STM/STS Investigations of Ag Nanoparticles Growth on Semiconductor Surfaces
}

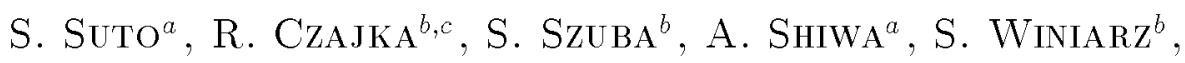 \\ H. NAgashima ${ }^{a}, \mathrm{H} \cdot \mathrm{KaTO}^{a}, \mathrm{~T} . \mathrm{YAMADA}^{d}$ AND A. KasuYa $^{c}$ \\ ${ }^{a}$ Department of Physics, Tohoku University, Sendai 980-8578, Japan \\ ${ }^{b}$ Faculty of Technical Physics, Poznan University of Technology \\ Nieszawska 13 A, 60-965 Poznań, Poland \\ ${ }^{c}$ Center for Interdisciplinary Research, Tohoku University \\ Sendai 980-8578, Japan
}

${ }^{d}$ Institute of Physical and Chemical Research, Wako 351-0198, Japan

We have investigated the growth of $\mathrm{Ag}$ nanoparticles deposited on $\mathrm{Si}(111), \mathrm{H} / \mathrm{Si}(111)-(1 \times 1)$ and $\mathrm{Bi}_{2} \mathrm{Te}_{3}$ substrates using a variable temperature scanning tunneling microscopy. These substrates are different as regards the model system for cluster and islands growth at the nanometer scale. Ag was evaporated onto the sample mounted at the scanning tunneling microscopy stage in vacuum of $10^{-10}$ Torr range during evaporation. The substrates were kept at different temperatures: $-150^{\circ} \mathrm{C}$, room temperature, and $300^{\circ} \mathrm{C}$ during the deposition process. In general, we have observed $3 \mathrm{D}$ growth mode up to several ML coverage. The density of clusters and their size were functions of the substrate's temperature during the deposition process - a higher density and a smaller size at $-150^{\circ} \mathrm{C}$ were in opposition to the $300^{\circ} \mathrm{C}$ results a lower density and a larger size. Low temperature depositions led to continuous layers above $10 \mathrm{ML}$ coverage but the surface was covered by small Ag clusters of $1-2 \mathrm{~nm}$ in heights and $2-3 \mathrm{~nm}$ in diameters. The log-log graphs of height and projected diameter of Ag clusters revealed different slopes indicating different growth mechanisms at low and high temperatures. We obtained the value of $n=0.25 \pm 0.02$, typical of the so-called droplet model of cluster growth, only at $300^{\circ} \mathrm{C}$. Scanning tunneling spectroscopy measurements revealed clearly different $I-V$ (and $\mathrm{d} I / \mathrm{d} V$ vs. bias voltage) curves measured above clusters and directly above the substrate. In discussion, we compared our results to theoretically calculated density of states from other papers, finding conformity for partial density of states.

PACS numbers: 68.37.Ef, 68.47.Fg, 68.55.Ac 


\section{Introduction}

It is a well-known fact that the epitaxy of thin metallic films is dependent on the early stage of nucleation [1]. Frequently, these films are composed of small discrete droplets (often named "clusters"), which grow, then coalesce into bigger droplets or islands, and finally create a complete layer. Both, the clusters and ultra thin films, exhibit new and specific physical properties due to their reduced dimensionality. Therefore, they have a wide and increasing number of scientific and commercial applications.

The substrates play a significant role in the film growth process. Semiconductors are mostly used as substrates to build integrated circuits. Si(100) or Si(111) represent substrates strongly interacting with adsorbents. This is a result of the presence of various dangling bonds at their surfaces [2]. Different passivation techniques were employed on silicon surfaces to make the surface less active and more uniform. One of such techniques is oxidation [3]. However, it is quite difficult to make the surface uniform as regards oxide thickness and its electronic properties. More recently, thin metallic nitride films have been used [4] to passivate the substrate or create a thin barrier layer before Cu plating, for example. One of the other methods is hydrogen termination using both the "dry method" in vacuum [5] and the so-called "wet method", by dipping the silicon substrate in HF or other solution containing both $\mathrm{H}$ and $\mathrm{F}$ ions $[6,7]$. The latter method gives a smooth surface with $1 \times 1$ reconstruction (as in bulk silicon) which weakly interacts with adsorbents. In this sense, substrate is closer to layered materials such as graphite (HOPG - highly oriented pyrolytic graphite), $\mathrm{MoS}_{2}$ or $\mathrm{Bi}_{2} \mathrm{Te}_{3}$. The last material has been attracting considerable attention due to its potential application in the micro-fabrication of integrated thermoelectric devices [8].

Transition from two-dimensional (2D) to three-dimensional (3D) growth during $\mathrm{Ag}$ or $\mathrm{Al} / \mathrm{Si}(111) 7 \times 7$ heteroepitaxy [9] is the main difference with the droplet growth on $\mathrm{H}-\mathrm{Si}(111) 1 \times 1$ or $\mathrm{Bi}_{2} \mathrm{Te}_{3}$ substrates followed by the transition to the fractal coalescence of small metallic clusters.

As regards thin layer's growth mechanisms during the early stage of the growth, it is generally accepted that the growth of clusters follows scaling laws such as the size $R(t) \sim t^{x}[10,11]$, where the exponent $x$ depends on the type of growth mechanism. For the growth of three-dimensional clusters the predicted value of $x$ is $1 / 4$ and for two-dimensional clusters $x$ is $1 / 2$. However, the experimental results of Zuo [12] and our results, presented in this paper, show that the small metallic clusters undergo stages of liquid-like rapid coalescence alternating with nucleation and growth. Step-like growth behavior was observed which deviates from the continuous time dependence of cluster density and sizes predicted by the scaling theory.

Scanning tunneling microscopy (STM) [13] and spectroscopy (STS) are one of the most suitable analytical techniques to visualize nanostructures in real space (with STM) and to investigate their specific electronic properties (with STS). 
The local density of electronic states (LDOS) can be measured using modulation techniques [14] or calculated [15] using current-voltage characteristics measured with the STM above the individual nanostructures or molecules or individual atoms. Quantum size effects (QSE) [16] or Coulomb blockade (CB) [17] effect may be observed as the sizes of small objects become comparable to the de Broglie wavelength of electrons confined within those objects. It was also shown that the STM/STS technique may be used for tracing both of the above-mentioned phenomena $[18,19]$.

In this paper we report our investigations of the growth of Ag nanoparticles deposited on $\mathrm{H}-\mathrm{Si}(111) 1 \times 1$ and $\mathrm{Bi}_{2} \mathrm{Te}_{3}$ substrates and measured at different temperatures: $-150^{\circ} \mathrm{C}(\mathrm{LT})$, room temperature $(\mathrm{RT})$, and $300^{\circ} \mathrm{C}(\mathrm{HT})$. These substrates are different as regards the model system for cluster and islands growth at the nanometer scale. The first one represents substrates adsorbing with strong forces and the latter two interact with weak forces on deposits [12]. We also present our current imaging tunneling spectroscopy (CITS) measurements as well as DOS maps calculated using the Bando method [15] with $I-V$ curves measured by us.

\section{Experimental}

Ag was evaporated from OMICRON EFM3 source onto the sample mounted at the STM stage in vacuum of $10^{-10}$ Torr range. The pressure during evaporation, although slightly worse, was kept in the same range. The commercial variable temperature STM enabled us to change the sample's temperature between $125 \mathrm{~K}$ (using liquid nitrogen) and $600 \mathrm{~K}$ using sample holder with built in the boron nitride (BN) heater.

The "exposure", defined as a product of evaporation time and ion flux, was our main parameter of the evaporation rate. The ion flux, which constitutes a small but constant part of the atomic flux, was measured by a circuit built into the evaporation source. Assuming that the ion flux was kept constant, the exposure was directly proportional to the evaporation time and could be used in our data analysis according to the scaling theory. We used fluxes between 20 and $200 \mathrm{nA} \mathrm{s}$ which gave us the deposition rates between 0.025 and $0.25 \mathrm{ML}$ per minute.

The $\mathrm{Si}(111) 7 \times 7$ substrate was prepared traditionally by flashing at about $1250^{\circ} \mathrm{C}$ for 10 seconds followed by the slow temperature fall between $900^{\circ} \mathrm{C}$ and $600^{\circ} \mathrm{C}$.

The H-Si(111) $1 \times 1$ substrates were prepared using the chemical "wet" method which consists of many cleaning procedure steps to remove $\mathrm{SiO}_{2}$ and finishes with the hydrogen termination in $40 \% \mathrm{NH}_{4} \mathrm{~F}$ solution. The samples were finally rinsed with clean (Millipore Quality) water and transferred to UHV chamber. Then, H-Si(111) samples were annealed at about $200^{\circ} \mathrm{C}$ temperature for several hours.

The $\mathrm{Bi}_{2} \mathrm{Te}_{3}$ substrates were cleaved using a scotch tape, immediately transferred to $\mathbf{U H V}$ chamber, and then annealed for 2 hours at about $200^{\circ} \mathrm{C}$. 
The sample's temperature during the STM measurements was kept at the same level as it was during the evaporation, for example if the deposition was done at $\mathrm{LT}\left(-150^{\circ} \mathrm{C}\right)$ also STM/STS measurements were done at the same temperature. We started with low exposures of $10^{3} \mathrm{nA}$ s range to finish with $10^{6}$ or $10^{7} \mathrm{nA} \mathrm{s}$. The sample in our setup was biased relatively to the tip. This means that the empty states were imaged for positive voltage, whereas the occupied ones were probed for negative bias. All STM topographic images were recorded in the constant current mode. We have used Ir cut tips as the STM probe.

\section{Results}

\subsection{STM topography images}

Figure 1 represents the STM images of substrates obtained with the atomic resolution. The image of $7 \times 7$ reconstruction on Si(111) shown in Fig. 1a is divided into two parts with negative and positive sample polarity, and illustrates the spatial distribution of highest occupied molecular orbital (HOMO) and lowest unoccupied molecular orbital (LUMO) states [2], respectively. Figure 1 b shows $1 \times 1$ reconstruction of $\mathrm{Si}(111)$ surface obtained due to hydrogen passivation during the chemical wet procedure [6]. One can easily see the fcc hexagonal type structure
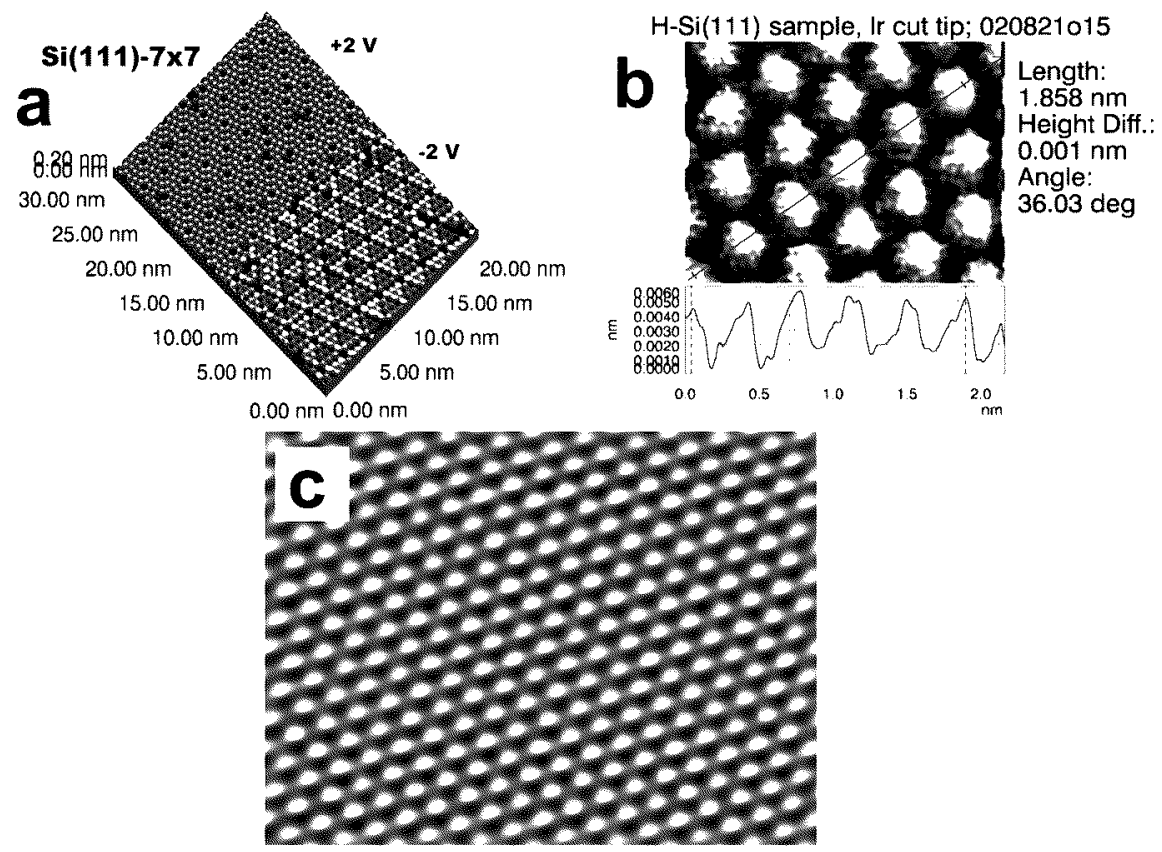

Fig. 1. STM images of: (a) $\mathrm{Si}(111) 7 \times 7$ substrate measured with positive bias (LUMO states) and negative bias (HOMO states); (b) H-passivated $\mathrm{Si}\left(111\right.$ ) substrate; (c) $\mathrm{Bi}_{2} \mathrm{Te}_{3}$ surface. 
with $0.38 \mathrm{~nm}$ lattice constant. However, a relatively high number of point (or bigger) defects could also be observed. Finally, Fig. 1c shows the atomic resolution image of tellurium atoms' plane unveiled after the $\mathrm{Bi}_{2} \mathrm{Te}_{3}$ sample cleavage. The measured lattice constant is $0.44 \mathrm{~nm}$ and it is close to X-ray data value of $0.438 \mathrm{~nm}$.

The typical images of silver clusters grown on $\mathrm{H}-\mathrm{Si}(111) 1 \times 1$ substrate at four different temperatures from $-150^{\circ} \mathrm{C}$ up to $300^{\circ} \mathrm{C}$ with the exposure of $2 \times 10^{4} \mathrm{nA}$ s are presented in Fig. 2. The amount of the Ag volume deposited on the substrate, in this case, was estimated with the help of WSxM software, and the values were $1.75 \pm 0.25 \mathrm{ML}, 0.75 \pm 0.05 \mathrm{ML}, 0.45 \pm 0.05 \mathrm{ML}$ and $0.35 \pm 0.05 \mathrm{ML}$ for substrate temperatures of $-150^{\circ} \mathrm{C}, \mathrm{RT}, 150^{\circ} \mathrm{C}$, and $300^{\circ} \mathrm{C}$, respectively. For coverage below $1 \mathrm{ML}$, we have observed cluster growth within the whole range of temperatures. In general, we have observed $3 \mathrm{D}$ growth mode up to several $\mathrm{ML}$ coverage. The density of clusters and their size were functions of the substrate's temperature during the deposition process - a higher density and a smaller size at LT was in opposition to the HT results - a lower density and a larger size. Qualitatively the same result, as regards the cluster density and its dimension one can observe in Fig. 3, where the STM images measured at LT, RT, and HT with the same deposited volume of $\mathrm{Ag}$ (1 ML and $5 \mathrm{ML}$ ) are collected. LT depositions led to continuous layers just above $10 \mathrm{ML}$ coverage but the surface was covered by small $\mathrm{Ag}$ clusters of $1-2 \mathrm{~nm}$ in heights and $2-3 \mathrm{~nm}$ in diameters.

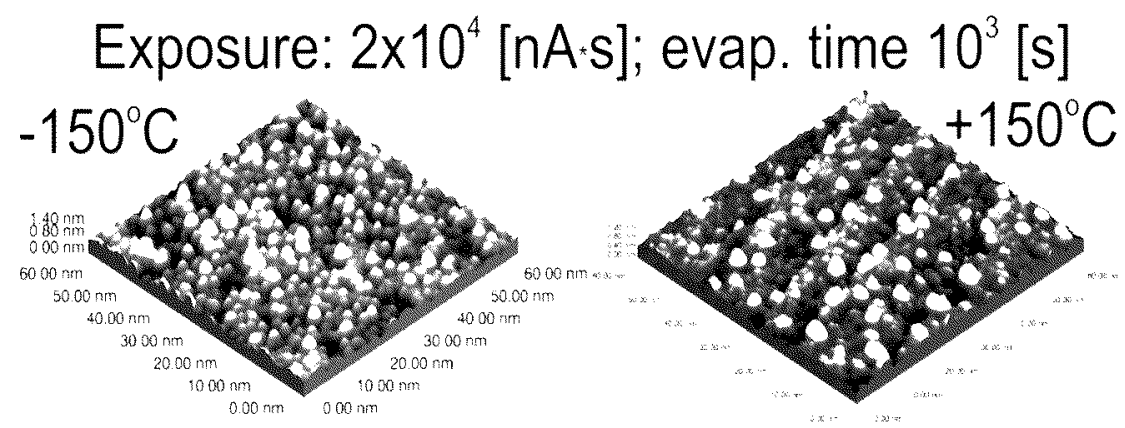

$64 \mathrm{~nm} \times 64 \mathrm{~nm}$

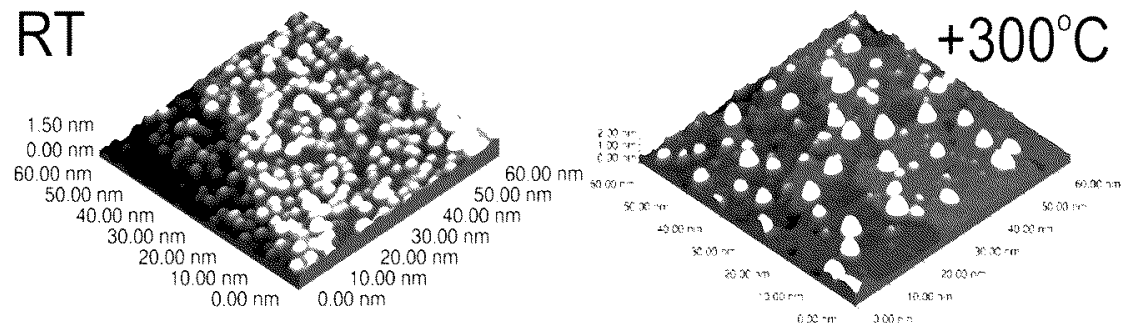

Fig. 2. The STM images of silver clusters grown on $\mathrm{H}-\mathrm{Si}(111) 1 \times 1$ substrate at four different temperatures from -150 up to $300^{\circ} \mathrm{C}$ with the exposure of $2 \times 10^{4} \mathrm{nA} \mathrm{s}$. 


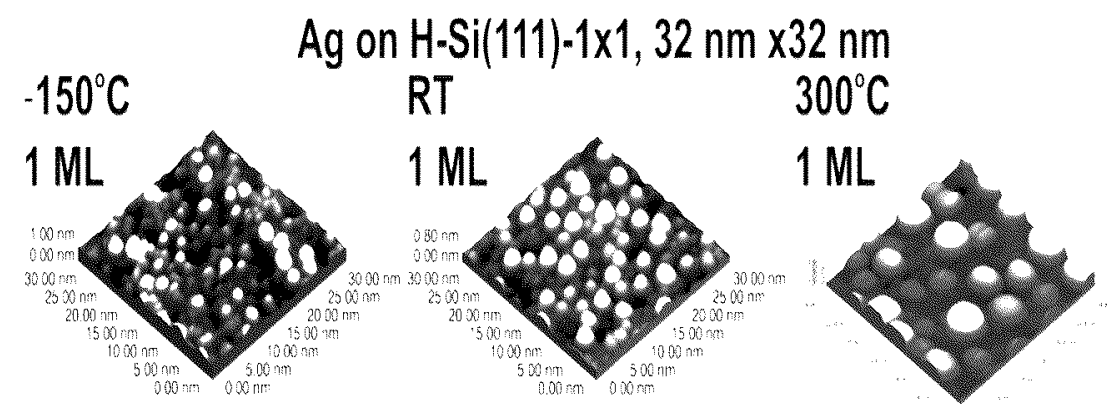

$5 \mathrm{ML}$

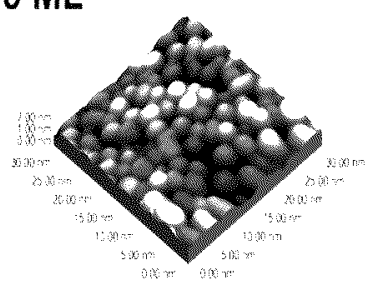

$5 \mathrm{ML}$

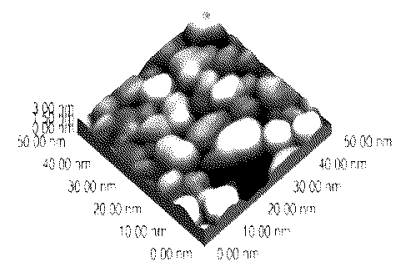

$5 \mathrm{ML}$

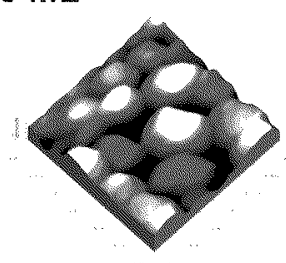

Fig. 3. The STM images measured at $-150^{\circ} \mathrm{C}$, RT, and $300^{\circ} \mathrm{C}$ with the same deposited volume of $\mathrm{Ag}$ ( $1 \mathrm{ML}$ - the top row, and $5 \mathrm{ML}$ - the bottom row).

\subsection{Clusters' diameter and height plots versus exposure}

The log-log graphs of height and projected diameter (as measured at the half of maximum height) of $\mathrm{Ag}$ clusters versus exposure were prepared to make the quantitative analysis of our data and compare our results with the predictions of the scaling theory $[10,11]$ and with the experimental data of other authors [12]. The obtained curves are presented in Fig. 4.

All the graphs of height and projected diameter of Ag clusters versus the exposure, besides the height plot at $300^{\circ} \mathrm{C}$, have revealed complicated structures. The plots at LT may be divided into two parts: below and above $3 \times 10^{5} \mathrm{nA} \mathrm{s}$ (or $5 \times 10^{3} \mathrm{nA}$ min as in Fig. 4's $x$-axis description). The apparent diameter is growing linearly in both ranges but with different slopes of $0.15 \pm 0.03$ and $0.30 \pm 0.08$, respectively (Fig. $4 \mathrm{a}$ and $\mathrm{b}$ ). The height plot has a bell-type shape below $3 \times 10^{5}$ nA s exposure and linear type with $0.52 \pm 0.08$ slope above. The plots at RT may be divided into three parts. Below $6 \times 10^{4} \mathrm{nA} \mathrm{s}\left(1 \times 10^{3} \mathrm{nA} \mathrm{min}\right)$ exposure the diameter fluctuates between 2 and $5 \mathrm{~nm}$, it becomes constant above this threshold up to $6 \times 10^{5} \mathrm{nA} \mathrm{s}\left(1 \times 10^{4} \mathrm{nA}\right.$ min $)$ exposure and it linearly increases afterwards with the slope of $0.59 \pm 0.06$. The height versus exposure plot changes in the same way but with different values. Finally, at $300^{\circ} \mathrm{C}$, the diameter is nearly constant $(3.5 \mathrm{~nm})$ up to $6 \times 10^{4} \mathrm{nA} \mathrm{s}\left(1 \times 10^{3} \mathrm{nA} \min \right)$ exposure and above it, it increases linearly with the slope of $0.21 \pm 0.04$. The height plot increases linearly within the whole range with the slope of $0.25 \pm 0.02$. Generally speaking, that data does not follow the scaling theory. 

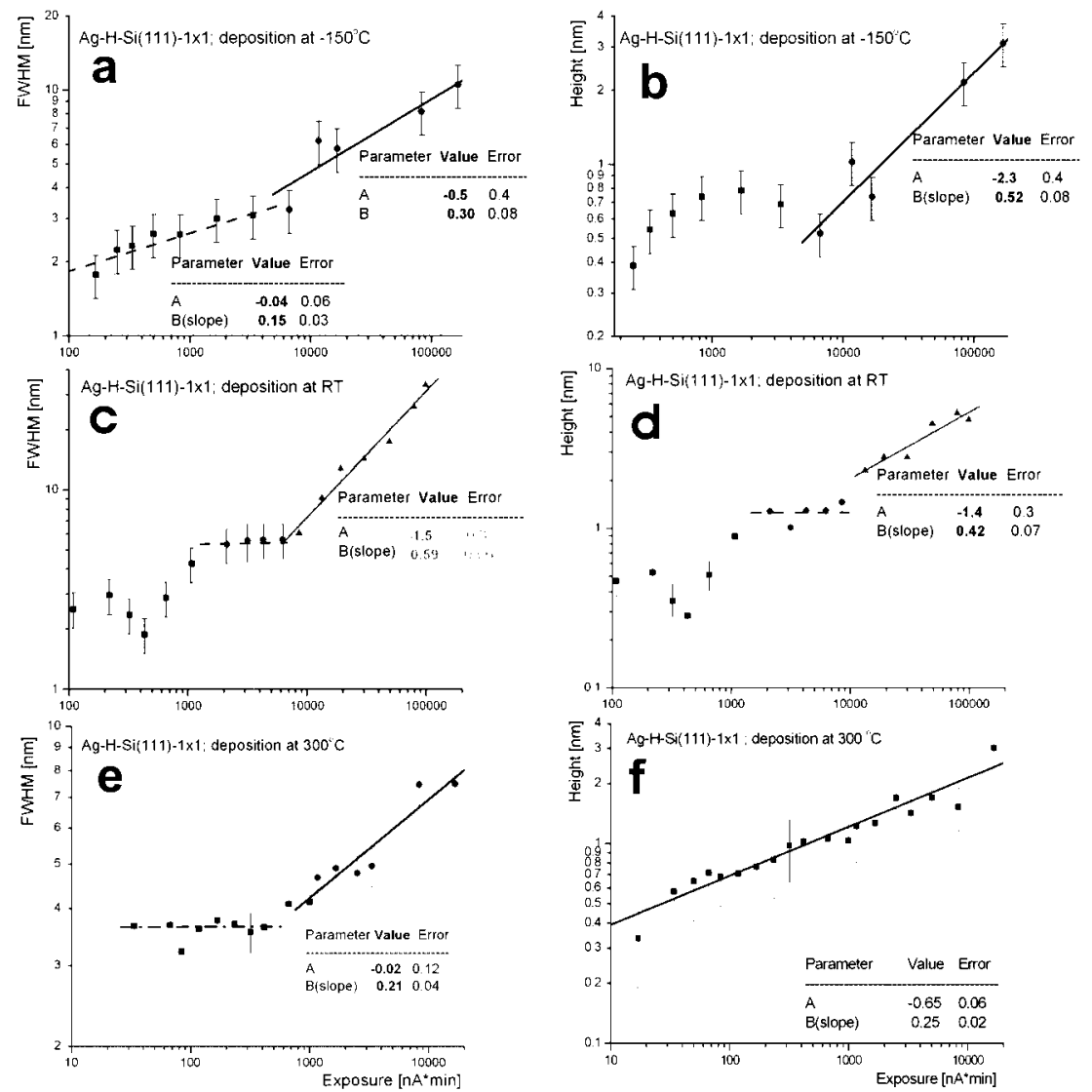

Fig. 4. The log-log graphs of height and projected diameter (as measured at the half of maximum height) of Ag clusters versus exposure (with [n A min] unit) measured at (a) and (b) $-150^{\circ} \mathrm{C},(\mathrm{c})$ and (d) RT and (e) and (f) $300^{\circ} \mathrm{C}$.

\subsection{Current imaging tunneling spectroscopy measurements}

Scanning tunneling spectroscopy measurements were performed at RT and at LT conditions. However, the LT measurements suffered a conductivity decrease in the semiconducting substrate which diminished the measurements' sensitivity around the Fermi level within -3 to +3 range, as it is shown in Fig. 5 .

Measurements at RT have revealed clearly different $I-V$ curves (and $\mathrm{d} I / \mathrm{d} V$ vs. bias voltage curves) measured above clusters and directly above the substrate. We performed current imaging tunneling spectroscopy (CITS) measurements, creating the tunnel current maps within a given range of bias voltage, from 1 up to a few volts, usually. The $I-V$ curves were measured at every point of the topographic image. Following these CITS measurements the density of states (DOS) was calculated from $I-V$ characteristics using the Bando method [15]. Figure 6 


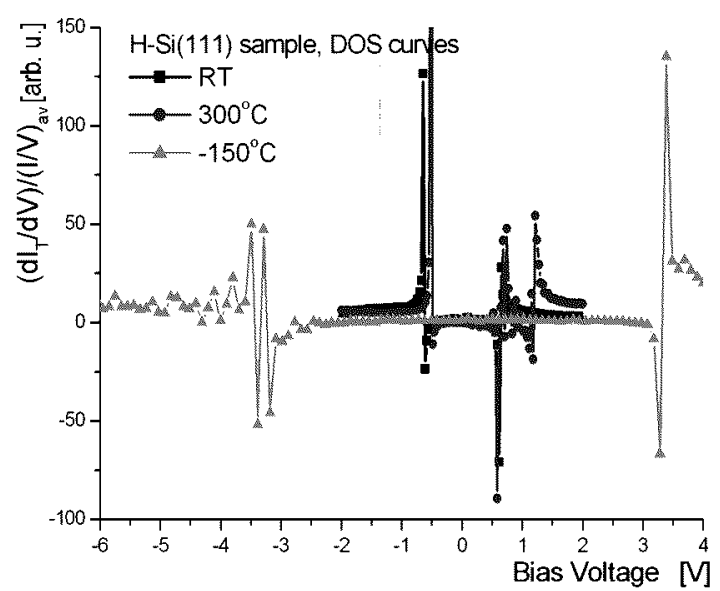

Fig. 5. The set of STS curves: $I-V, \mathrm{~d} I / \mathrm{d} V$, and $(\mathrm{d} I / \mathrm{d} V) /(I / V)_{\mathrm{av}}$ vs. bias voltage measured above clean $\mathrm{H}-\mathrm{Si}(111) 1 \times 1$ at LT, RT, and HT conditions.

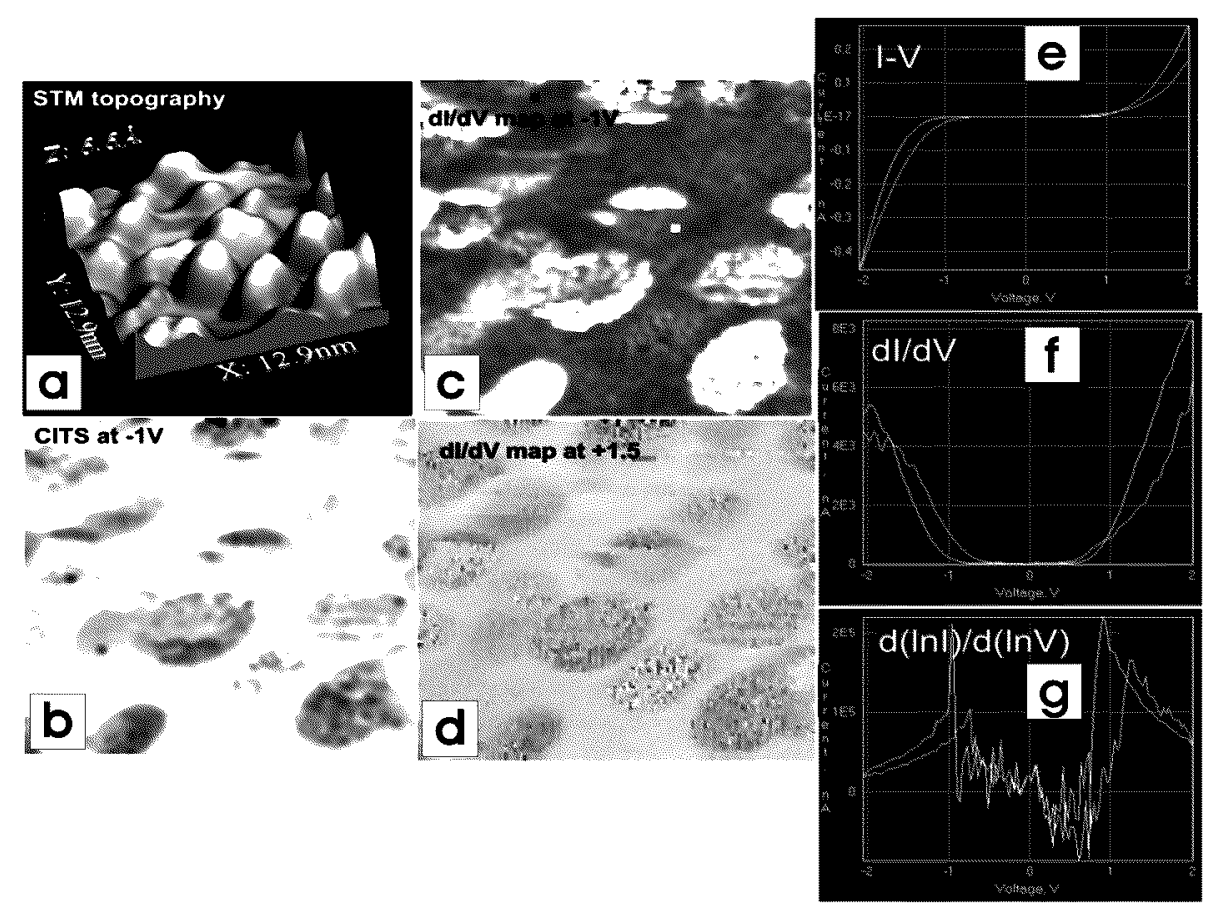

Fig. 6. The set of CITS results obtained on Ag clusters deposited on H-Si(111) $1 \times 1$ substrate: STM topography image (a), tunnel current map at $-1 \mathrm{~V}$ bias polarization (b), the calculated spatial DOS distribution at the surface measured with two different bias polarizations $-1 \mathrm{~V}$ (c) and $+1.5 \mathrm{~V}(\mathrm{~d}), I-V$ curves (e), $\mathrm{d} I / \mathrm{d} V$ vs. bias voltage curve (f), and DOS vs. bias voltage (or energy) calculated using the Bando method (see the footnote on p. ) (g). 
shows a typical set of CITS results including topography image (Fig. 6a), tunnel current map at given bias polarization (Fig. 6b), spatial DOS distribution at the surface at two different bias polarizations (Fig. $6 \mathrm{c}$ and d), $I-V$ curves (Fig. 6e), $\mathrm{d} I / \mathrm{d} V$ vs. bias voltage curve (Fig. 6f), and DOS vs. bias voltage (or energy) calculated using the Bando method* (Fig. 6g).

\section{Discussion}

\subsection{Clusters' growth}

The difference between the $\mathrm{Ag}$ or $\mathrm{Al}$ structures on $\mathrm{Si}(111)$ and $\mathrm{H}-\mathrm{Si}(111)$ is distinct due to the strong and weak interactions with adsorbents on both substrates, respectively. For example, it is a well-known result that $\mathrm{Ag}$ on $\mathrm{Si}(111)$ creates the wetting layer first (with $\sqrt{3} \times \sqrt{3}$ reconstruction) and small islands then grow at the top of this layer [20]. We shall skip the Ag growth on $\mathrm{Si}(111)$ as it was discussed in many publications and we shall concentrate on our Ag cluster growth on H-passivated silicon.

The interaction between $\mathrm{Ag}$ and $\mathrm{H}$ terminated and therefore fully bonded $\mathrm{Si}$ is weak and Volmer-Weber (3-dimensional) growth of clusters is dominating as it is shown in Fig. 2. This cluster growth is a result of reduced, due to H-passivation, surface and interface energies. The $\mathrm{Bi}_{2} \mathrm{Te}_{3}$ substrate also belongs to lower energy surfaces. Therefore, nanometer-sized Ag clusters grow in droplet-like mode as it is shown in Fig. 7. This occurs when the binding energy of the condensate atoms or molecules to each other is greater than their binding energy to the substrate atoms.

According to the scaling laws the 3D cluster growth should scale as $R(t) \sim t^{x}$, with $x=1 / 4$ and $x=1 / 2$ in the case of $2 \mathrm{D}$ island growth. Our results, presented in Fig. 3 , show that for short times of the clusters' growth or in the small size regime these scaling laws are not followed. These results are qualitatively in agreement with Zuo et al. TEM investigations [12]. At LT, the clusters' growth during the nucleation/growth stage follows the power law $R(t) \sim t^{x}$, with $x=0.15$ up to $3.0 \times 10^{5} \mathrm{nA}$ s exposure, so well below the expected 0.25 value from the scaling law, most probably due to decreased diffusion of atoms at the surface. However, the $x$ value increases up to 0.30 , which is close to 0.25 value within the experimental error, above $3.0 \times 10^{5} \mathrm{nA} \mathrm{s}$ exposure. This would suggest a smaller diffusion barrier of $\mathrm{Ag}$ on $\mathrm{Ag}$ than $\mathrm{Ag}$ on $\mathrm{H}-\mathrm{Si}(111)$ surface at LT, as for this exposure nearly the whole surface is covered by Ag material. The height of clusters increases up to 3 monoatomic layers and reaches a maximum at about $1.5 \times 10^{5} \mathrm{nA}$ s exposure, then it decreases to 2 monoatomic layers most probably due to secondary adsorption of $\mathrm{Ag}$ between existing clusters. The clusters' height increases again above $3.0 \times 10^{5} \mathrm{nA}$ s exposure, however with the slope of 0.5 which is even higher than the slope for diameter and results in $3 \mathrm{D}$ growth of $\mathrm{Ag}$ clusters.

* The software was elaborated by S. Szuba. 


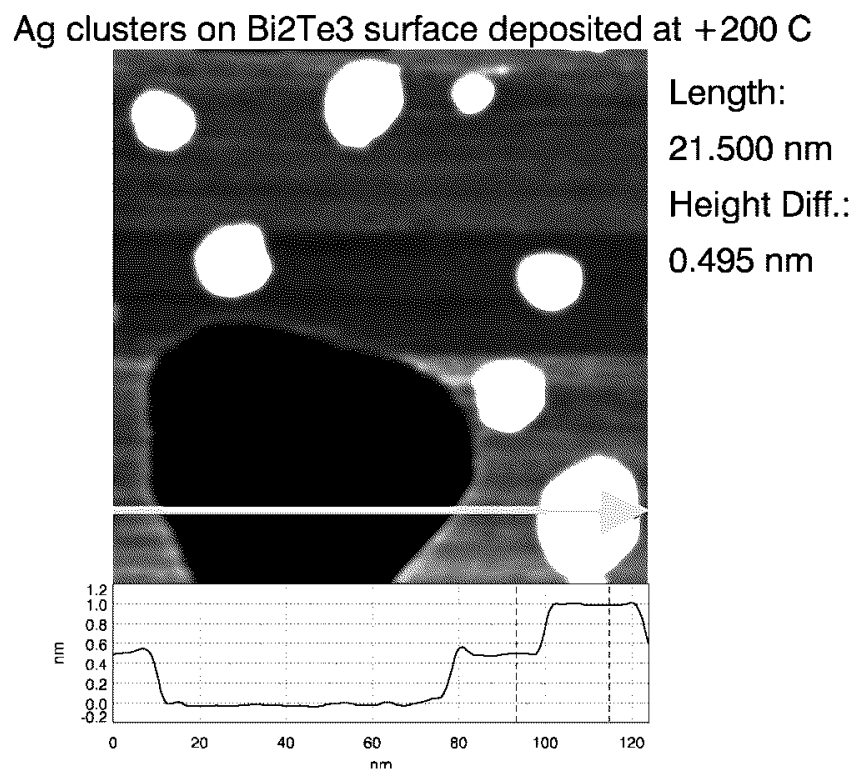

Fig. 7. The STM images of silver clusters grown on the $\mathrm{Bi}_{2} \mathrm{Te}_{3}$ substrate at $200^{\circ} \mathrm{C}$. At the bottom - cross line section measured along the line marked at the topography image.

The clusters' diameter and height fluctuate up to $6 \times 10^{4} \mathrm{nA}$ s exposure (about $1 \mathrm{ML}$ of the deposited material) at RT on the level of $3 \mathrm{~nm}$ and $0.5 \mathrm{~nm}$, respectively. These values become, respectively, $(5 \pm 1) \mathrm{nm}$ and $(1.25 \pm 0.20) \mathrm{nm}$ for exposures up to $6 \times 10^{5} \mathrm{nA} \mathrm{s}$ (up to $4 \mathrm{ML}$ ) while the density of clusters falls down. That means that the step-like growth of the Ag droplets took place at this range of exposures. Similar processes were observed for the diameter of a single droplet grown due to coalescence, as it was studied by Beysens et al. [21]. Above the threshold of $6 \times 10^{5} \mathrm{nA}$ s exposure the diameter grows with the slope $0.59 \pm 0.06$ which is close to 0.50 value, typical of $2 \mathrm{D}$ island growth. That means that the late stage of growth takes place above the deposition of $4 \mathrm{ML}$ of $\mathrm{Ag}$ at RT.

The diameter of $\mathrm{Ag}$ clusters is kept constant on the level of $3.6 \pm 0.3 \mathrm{~nm}$ at high temperature conditions $\left(300^{\circ} \mathrm{C}\right)$ while the height and density of clusters per area unit are increasing. This fact would suggest that clusters of constant and preferable diameter of $3.6 \mathrm{~nm}$ are created while their height increases from 2 up to 4 monoatomic thickness. The 3D growth of $\mathrm{Ag}$ clusters takes place above the threshold of $6 \times 10^{4} \mathrm{nA}$ s exposure and both diameter and height increase with the slope close to or exactly $0.25 \mathrm{~nm}$ which is in agreement with the scaling law for this type of growth.

The model of the droplet growth may be divided into early and late stage regimes. The first one includes the formation of the first stable nuclei. Nearly all clusters have a smaller equilibrium concentration than supersaturation and thus 
grow [10]. The cluster size distribution changes as a function of radius and time. During the late stage large clusters grow at the expense of smaller ones. This process is known as Ostwald ripening. According to this model the transition from early to late stage influences the cluster size distribution. Our experimental data received at HT conditions fit qualitatively the theoretical curves as it is seen in Fig. 8, where cluster size distribution diagrams corresponding to $0.2 \mathrm{ML}$ and $5 \mathrm{ML}$ deposition of Ag material are shown. Figure $8 \mathrm{~b}$ reproduces the theoretical curves derived from Fick's second law of diffusion and Gibbs-Thomas equation [10].
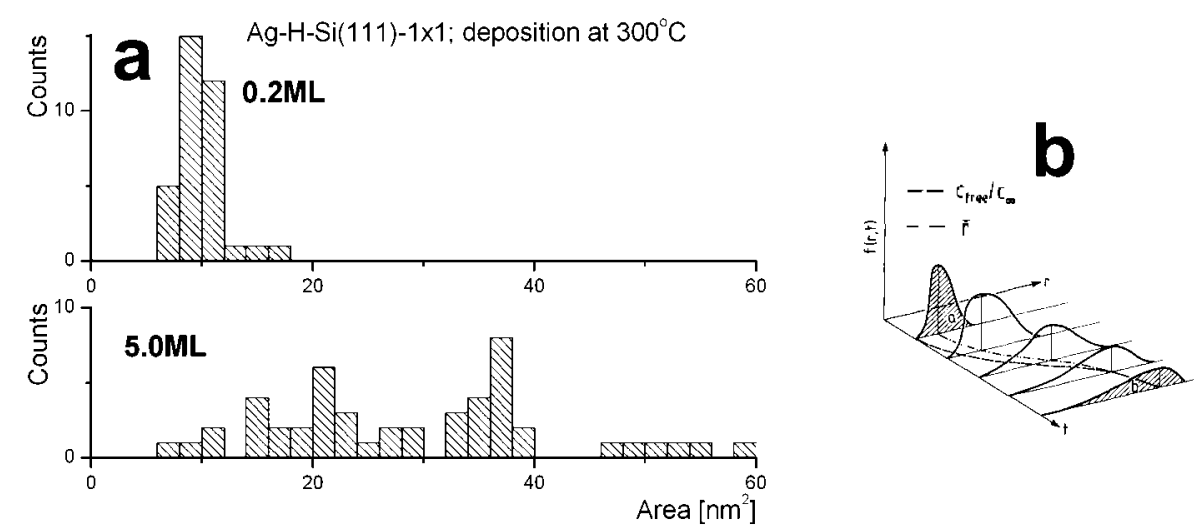

Fig. 8. (a) Clusters' size distribution diagrams corresponding to $0.2 \mathrm{ML}$ and $5 \mathrm{ML}$ deposition of Ag material. (b) The theoretical curves derived from Fick's second law of diffusion and Gibbs-Thomas equation [23].

Summarizing this part, we can say that we have found close agreement between the scaling law of early stage clusters' growth and experimentally observed data only at HT conditions.

\subsection{Scanning tunneling spectroscopy on clusters}

STM construction enables STS-type measurements by stopping the scanning at every or at any desired point on the investigated surface, switching off the feedback loop and performing the $I-V$ characteristics measurements. One may calculate or measure, using the modulation techniques, the dynamic conductivity ( $\mathrm{d} I / \mathrm{d} V$ vs. bias polarization) which is more or less straightforwardly related to the local density of electronic states (LDOS) [22].

The electron charge density is explicitly present in the tunnel current formula as follows:

$$
I \propto \int_{0}^{e V} \rho_{\mathrm{s}}(r, E) \rho_{\mathrm{t}}(r,-e V+E) T(E, e V, r) \mathrm{d} E,
$$

where $\rho_{\mathrm{s}}(r, E)$ and $\rho_{\mathrm{t}}(r, E)$ represent density of states for the sample and probe, respectively, evaluated at position $r$ and for energy $E$ measured against their in- 
dividual Fermi's levels. $T(E, e V, r)$ is tunnel transmission probability for electrons with energy $E$ at given bias polarization

$$
T(E, e V)=\exp \left(-\frac{2 s \sqrt{2 m}}{\hbar} \sqrt{\frac{\phi_{\mathrm{s}}+\phi_{\mathrm{t}}}{2}+\frac{e V}{2}-E}\right),
$$

where $s$ - sample-probe distance, $\phi_{\mathrm{s}}$ and $\phi_{\mathrm{t}}$ - work functions for the sample and probe, respectively, $\hbar$ - Planck's constant $/ 2 \pi$.

Usually DOS for metal tips (W, Pt/Ir, Au) are a smooth and continuous function of energy (or bias voltage), therefore any peculiarities seen in STM images must have originated in sample. One can expect such peculiarities when imaging small clusters or any other low-dimensional structures (nanowires, quantum dots). We have used the so-called Bando method [15] to calculate the LDOS from $I-V$ measurements and create maps of the spatial distribution of LDOS. The individual $I-V$ curves measured at the same point are noisy with some accidental jumps due to the extreme sensitivity of STM to vibration, and due to the fact that during $I-V$ measurements the feedback loop, which controls the STM tip-sample distance, is switched off. Therefore, presented here $I-V$ curves are averaged over few tens or even thousands of curves, usually. The LDOS maps were created using software developed by one of co-authors - S. Szuba.

Figure 5 shows a set of STS curves: $I-V, \mathrm{~d} I / \mathrm{d} V$, and $(\mathrm{d} I / \mathrm{d} V) /(I / V)$ av vs. bias voltage measured above clean $\mathrm{H}-\mathrm{Si}(111) 1 \times 1$ at LT, RT, and HT conditions. The DOS curves revealed the energy gap which is clearly seen (pronounced peaks) at $\mathrm{RT}$ and whose value is about $1.35 \mathrm{eV}$. The energy gap at $300^{\circ} \mathrm{C}$ was kept very close to the RT one $(1.31 \mathrm{eV})$ which confirms that hydrogen termination is kept at this temperature. However, some additional states above the $0.63 \mathrm{eV}$ and $1.22 \mathrm{eV}$ appeared on the sample's occupied states side, with the highest one at $1.22 \mathrm{eV}$. We could observe the first strong DOS lines at LT conditions below $-3.3 \mathrm{eV}$ and above $+3.3 \mathrm{eV}$ due to decreased conductivity of semiconductor sample at LT. This fact limited our ability to investigate low energy (vs. Fermi energy) DOS states. The lines above $3.3 \mathrm{eV}$ on both sides of Fermi energy (FE) level may be related to the emission from surface states and/or surface resonance as it was found by Arranz et al. [23] using photoelectron spectroscopy. However, the threshold is moved towards FE level by about $0.7 \mathrm{eV}$ and we cannot exclude emission from the substrate related to the bulk Si transitions.

Figure 6 represents a typical set of our CITS data measured for Ag clusters grown on $\mathrm{H}-\mathrm{Si}(111) 1 \times 1$ substrate after $\mathrm{Ag}$ exposure of $7 \times 10^{3} \mathrm{nA}$ s at RT. The STM topography (Fig. 6a) reveals the presence of several clusters of $2 \mathrm{~nm}$ in average diameter. The current map (CITS) exhibits contrast between clusters of different sizes and the substrate (Fig. 6b). The DOS curves reveal the presence of energy gap of about $1.4 \mathrm{eV}$ for the substrate and smaller clusters. The bigger clusters show an apparent gap of $0.8 \mathrm{eV}$. The smaller clusters are mostly 1 monoatomic layer high and the bigger ones are of two or three atomic layers in height. This fact would suggest that only double atomic or thicker clusters may 
change the width of the observed gap due to the local electron charge transfer to the substrate. The calculated DOS map, using the Bando method, also reveals a similar type of contrast. This contrast changes with energy and one can observe the enhanced density of occupied states at clusters spots (e.g. at $-1.0 \mathrm{eV}$ ) - Fig. 6c, and reduced density of states on empty states' side (e.g. at $+1.7 \mathrm{eV})-$ Fig. 6 d. Figures $6 \mathrm{e}^{-} \mathrm{g}$ represent $I-V, \mathrm{~d} I / \mathrm{d} V, \mathrm{~d}(\ln I) / \mathrm{d}(\ln V)$ vs. bias polarization curves and cross line profile of DOS map at above-mentioned bias polarization values, respectively. The $I-V$ and other spectroscopic curves evaluate towards metallic type curves with the increasing Ag exposures.

CITS maps measured over the clean $\mathrm{Bi}_{2} \mathrm{Te}_{3}$ surface exhibited a contrast between different terraces or islands. The $\mathrm{d} I / \mathrm{d} V(\sim \mathrm{DOS})$ curves referred to those planes were compared with theoretically calculated DOS. We could conclude that curve measured above one of terraces is similar to DOS of Bi $p$ orbital presented in Ref. [24]. Adequately the curve measured above different terraces resembles the DOS curve of Te-1 $p$ orbital. This analysis allowed us to distinguish between bismuth and tellurium planes. The detailed analysis and data are reported by Winiarz et al. [25] in the same issue of Acta Physica Polonica A.

\section{Conclusions}

The growth of Ag clusters deposited on $\mathrm{Si}(111) 7 \times 7$ surface for small exposures depends strongly on the substrate temperature. The density of clusters was higher and the size was smaller at LT in opposition to the HT results - a lower density and a larger cluster's size. The log-log graphs of height and projected diameter of $\mathrm{Ag}$ clusters revealed different slopes (between 0.25 to 0.59 ) indicating different growth mechanisms at low and high temperatures. We obtained the value of $n=0.25 \pm 0.02$, typical of the so-called droplet model of cluster growth, only at $300^{\circ} \mathrm{C}$. The density of clusters was much smaller and their diameters were much bigger when the Ag clusters were grown on weakly interacting substrates as $\mathrm{Bi}_{2} \mathrm{Te}_{3}$.

Scanning tunneling spectroscopy measurements revealed clearly different $I-V$ curves (and $\mathrm{d} I / \mathrm{d} V$ vs. bias voltage curves) measured above clusters and directly above the substrate. The $I-V$ and other spectroscopic curves evolve from semiconductor type towards metallic type curves with increasing Ag thickness (above $2 \mathrm{ML}$ ).

CITS maps measurements enabled the evaluation of LDOS from $I-V$ characteristics using the Bando method. The created maps of LDOS distribution showed variation of the LDOS with nanometer spatial resolution.

\section{Acknowledgments}

As regards Polish co-authors, this work was supported by the Ministry of Scientific Research and Information Technology (Project No. 2P03B 042 25). 


\section{References}

[1] D. Walton, J. Chem. Phys. 37, 2182 (1962).

[2] K. Takayanagi, Y. Tanishiro, M. Takahashi, S. Takahashi, J. Vac. Sci. Technol. A 3, 1502 (1985).

[3] G.D. Wilk, Yi Wei, Hal Edwards, R.M. Wallace, Appl. Phys. Lett. 70, 2288 (1997).

[4] R.N. Wang, J.Y. Feng, Y. Huang, Appl. Surf. Sci. 207, 139 (2003).

[5] D. Gräf, M. Grundner, R. Schulz, L. Mühlhoff, J. Appl. Phys. 68, 5155 (1990).

[6] G.S. Higashi, Y.J. Chabal, G.W. Trucks, K. Raghavachari, Appl. Phys. Lett. 56, $656(1990)$.

[7] T. Yamada, T. Inoue, K. Yamada, N. Takano, T. Osaka, H. Harada, K. Nishiyama, I. Taniguchi, J. Am. Chem. Soc. 125, 8039 (2003).

[8] Gao Min, D.M. Rowe, Solid State Electron. 43, 923 (1999).

[9] P. Sobotik, I. Ostadal, J. Myslivecek, T. Jarolimek, F. Lavicky, Surf. Sci. 482-485, 797 (2001)

[10] M. Zinke-Allmang, L.C. Feldman, M.H. Grabov, Surf. Sci. Rep. 16, 377 (1992).

[11] F. Family, P. Meakin, Phys. Rev. Lett. 61, 428 (1988).

[12] J.M. Zuo, B.Q. Li, Phys. Rev. Lett. 88, 255502 (2002).

[13] G. Binnig, H. Rohrer, Helv. Phys. Acta 55, 726 (1982).

[14] R.J. Hamers, R.M. Tromp, J.E. Demuth, Phys. Rev. Lett. 56, 1972 (1986).

[15] H. Bando, T. Shimitzu, Y. Aiura, Y. Haruyama, K. Oka, Y. Nishihara, J. Vac. Sci. Technol. B 14, 1060 (1996).

[16] H. Frohlich, Physica 4, 406 (1937).

[17] D.V. Averin, K.K. Likharev, J. Low Temp. Phys. 62, 345 (1986).

[18] J.G.A. Dubois, J.W. Gerritsen, S.E. Shafranjuk, E.J.G. Boon, G. Schmid, H. van Kempen, Europhys. Lett. 33, 279 (1996).

[19] P.J.M. van Bentum, R.T.M. Smokers, H. van Kempen, Phys. Rev. Lett. 60, 2543 (1988).

[20] K.J. Wan, X.F. Lin, J. Nogami, Phys. Rev. B 47, 13700 (1992).

[21] D. Beysens, C.M. Knobler, Phys. Rev. Lett. 57, 1433 (1986).

[22] R.M. Feenstra, J.A. Stroscio, A.P. Fein, Surf. Sci. 181, 295 (1987).

[23] A. Arranz, J.F. Sanchez-Royo, J. Avila, V. Perez-Dieste, P. Dumas, M.C. Asensio, Phys. Rev. B 65, 075405 (2002)

[24] P. Larson, S.D. Mahanti, Phys. Rev. B 61, 8162 (2000).

[25] S. Winiarz, R. Czajka, S. Suto, P. Lośtak, S. Szuba, A. Kasuya, Acta Phys. Pol. A 104, (2003). 